\title{
Asymptotic Stabilization by State Feedback for a Class of Stochastic Nonlinear Systems with Time-Varying Coefficients
}

\author{
Hui Wang, 1 Wuquan Li, ${ }^{1,2}$ and Xiuhong Wang1 \\ ${ }^{1}$ School of Mathematics and Statistics Science, Ludong University, Yantai 264025, China \\ ${ }^{2}$ Key Laboratory of Systems and Control, Institute of Systems Science, Academy of Mathematics and Systems Science, \\ Chinese Academy of Sciences, Beijing 100190, China
}

Correspondence should be addressed to Wuquan Li; sea81@126.com

Received 16 December 2013; Accepted 31 January 2014; Published 11 March 2014

Academic Editor: Xuejun Xie

Copyright (C) 2014 Hui Wang et al. This is an open access article distributed under the Creative Commons Attribution License, which permits unrestricted use, distribution, and reproduction in any medium, provided the original work is properly cited.

\begin{abstract}
This paper investigates the problem of state-feedback stabilization for a class of upper-triangular stochastic nonlinear systems with time-varying control coefficients. By introducing effective coordinates, the original system is transformed into an equivalent one with tunable gain. After that, by using the low gain homogeneous domination technique and choosing the low gain parameter skillfully, the closed-loop system can be proved to be globally asymptotically stable in probability. The efficiency of the state-feedback controller is demonstrated by a simulation example.
\end{abstract}

\section{Introduction}

Consider a class of upper-triangular stochastic nonlinear systems with time-varying control coefficients described by

$$
\begin{gathered}
d x_{1}=\left(d_{1}(t) x_{2}+f_{1}\left(\tilde{x}_{3}\right)\right) d t+g_{1}^{T}\left(\tilde{x}_{3}\right) d \omega, \\
d x_{2}=\left(d_{2}(t) x_{3}+f_{2}\left(\tilde{x}_{4}\right)\right) d t+g_{2}^{T}\left(\tilde{x}_{4}\right) d \omega, \\
\vdots \\
d x_{n-2}=\left(d_{n-2}(t) x_{n-1}+f_{n-2}\left(\tilde{x}_{n}\right)\right) d t+g_{n-2}^{T}\left(\tilde{x}_{n}\right) d \omega, \\
d x_{n-1}=d_{n-1}(t) x_{n} d t, \\
d x_{n}=d_{n}(t) u d t,
\end{gathered}
$$

where $x=\left(x_{1}, \ldots, x_{n}\right)^{T} \in \mathbb{R}^{n}, u \in \mathbb{R}$ are the measurable state and the input of system, respectively. $\tilde{x}_{i}=\left(x_{i}, \ldots, x_{n}\right)^{T}$. $\omega$ is an $r$-dimensional standard Wiener process defined on a probability space $(\Omega, \mathscr{F}, P)$, with $\Omega$ being a sample space, $\mathscr{F}$ being a filtration, and $P$ being a probability measure. The functions $f_{i}: \mathbb{R}^{n-i-1} \rightarrow \mathbb{R}$ and $g_{i}: \mathbb{R}^{n-i-1} \rightarrow \mathbb{R}^{r}, i=$ $1, \ldots, n-2$, are assumed to be $\mathscr{C}^{1}$ with their arguments and $f_{i}(0)=0, g_{i}(0)=0 . d_{i}: R_{+} \rightarrow R, i=1, \ldots, n$, are unknown time-varying control coefficients with known sign.

In recent years, the global controller design for stochastic nonlinear systems has been attracting more and more attention. According to the difference of selected Lyapunov functions, the existing literature on controller design can be mainly divided into two types. One type is to derive the backstepping controller design by using quadratic Lyapunov function and a risk-sensitive cost criterion [1-3]. Another essential improvement belongs to Krstić and Deng. By introducing the quartic Lyapunov function, [4-12] present asymptotical stabilization control under the assumption that the nonlinearities equal zero at the equilibrium point of the open-loop system. Subsequently, for several classes of stochastic high-order nonlinear systems, by combining Krstić and Deng's method with stochastic analysis, $[13,14]$ study the problem of state-feedback stabilization and the outputfeedback stabilization problem is considered in $[15,16]$.

The study of stabilization control for upper-triangular nonlinear systems has long been recognized as difficult due to the inherent nonlinearity. In the existing literature, most results are established using the nested-saturation method $[17,18]$ and forwarding technique [19]. When no a priori 
information of the system nonlinearities is known, the work [20] proposes a universal stabilizer for feedforward nonlinear systems by employing a switching controller. Note that the listed results above do not consider the stochastic noise. However, from both practical and theoretical points of view, it is more important to study the control of uppertriangular stochastic nonlinear systems with time-varying control coefficients. Therefore, in this paper, under some appropriate assumptions, we consider the stabilization for system (1). To the best of the authors' knowledge, there are not any results about this topic.

In this paper, based on the low gain homogeneous domination technique, for system (1), we design a stabilization state-feedback controller, under which the closed-loop systems can be proved to be globally asymptotically stable in probability.

The contributions of this paper are highlighted as follows.

(i) This paper is the first result about state-feedback stabilization of upper-triangular stochastic nonlinear systems with time-varying control coefficients.

(ii) Due to the complex of upper-triangular system structure, how to deal with stochastic noise and timevarying control coefficients in the controller design is a nontrivial work.

The remainder of this paper is organized as follows. Section 2 offers some preliminary results. The state-feedback controller is designed and analyzed in Section 3. After that, in Section 4, a simulation example is presented to show the effectiveness of the state-feedback controller. Finally, the paper is concluded in Section 5.

\section{Preliminary Results}

The following notation will be used throughout the paper. $\mathbb{R}_{+}$ denotes the set of all nonnegative real numbers. For a given vector or matrix $X, X^{T}$ denotes its transpose, $\operatorname{Tr}\{X\}$ denotes its trace when $X$ is square, and $|X|$ is the Euclidean norm of a vector $X . \mathscr{C}^{i}$ denotes the set of all functions with continuous $i$ th partial derivatives. $\mathscr{K}$ denotes the set of all functions: $\mathbb{R}_{+} \rightarrow \mathbb{R}_{+}$, which are continuous, strictly increasing, and vanishing at zero; $\mathscr{K}_{\infty}$ denotes the set of all functions which are of class $\mathscr{K}$ and unbounded; $\mathscr{K} \mathscr{L}$ denotes the set of all functions $\beta(s, t): \mathbb{R}_{+} \times \mathbb{R}_{+} \rightarrow \mathbb{R}_{+}$, which are of $\mathscr{K}$ for each fixed $t$ and decrease to zero as $t \rightarrow \infty$ for each fixed $s$.

Consider the following stochastic nonlinear system:

$$
d x=f(x) d t+g^{T}(x) d \omega
$$

where $x \in \mathbb{R}^{n}$ is the state of the system and $\omega$ is an $r$ dimensional standard Wiener process defined on the probability space $(\Omega, \mathscr{F}, P)$. The Borel measurable functions $f$ : $\mathbb{R}^{n} \rightarrow \mathbb{R}^{n}$ and $g^{T}: \mathbb{R}^{n} \rightarrow \mathbb{R}^{n \times r}$ are local Lipschitz in $x \in \mathbb{R}^{n}$.

The following definitions and lemma will be used throughout the paper.
Definition 1 (see [5]). For any given $V(x) \in \mathscr{C}^{2}$ associated with stochastic system (2), the differential operator $\mathscr{L}$ is defined as

$$
\mathscr{L} V(x) \triangleq \frac{\partial V(x)}{\partial x} f(x)+\frac{1}{2} \operatorname{Tr}\left\{g(x) \frac{\partial^{2} V(x)}{\partial x^{2}} g^{T}(x)\right\} .
$$

Definition 2 (see [5]). For the stochastic system (2) with $f(0)=0, g(0)=0$, the equilibrium $x(t)=0$ of $(2)$ is globally asymptotically stable (GAS) in probability if, for any $\varepsilon>0$, there exists a class $\mathscr{K} \mathscr{L}$ function $\beta(\cdot, \cdot)$ such that $P\left\{|x(t)|<\beta\left(\left|x_{0}\right|, t\right)\right\} \geq 1-\varepsilon$ for any $t \geq 0$ and $x_{0} \in \mathbb{R}^{n} \backslash\{0\}$.

Lemma 3 (see [5]). Consider the stochastic system (2); if there exist a $\mathscr{C}^{2}$ function $V(x)$, class $\mathscr{K}_{\infty}$ functions $\alpha_{1}$ and $\alpha_{2}$, constants $c_{1}>0$ and $c_{2} \geq 0$, and a nonnegative function $W(x)$ such that

$$
\alpha_{1}(|x|) \leq V(x) \leq \alpha_{2}(|x|), \quad \mathscr{L} V \leq-c_{1} W(x)+c_{2},
$$

then

(a) for (2), there exists an almost surely unique solution on $[0, \infty)$;

(b) when $c_{2}=0, f(0)=0, g(0)=0$, and $W(x)=\alpha_{3}(|x|)$, where $\alpha_{3}(\cdot)$ is a class $\mathscr{K}$ function, then the equilibrium $x=0$ is GAS in probability and $P\left\{\lim _{t \rightarrow \infty}|x(t)|=\right.$ $0\}=1$.

\section{Controller Design and Stability Analysis}

The following assumptions are made on system (1).

Assumption 1. For $i=1, \ldots, n$, there exists a constant $b>0$ such that

$$
\begin{aligned}
& \left|f_{i}\left(\tilde{x}_{i+2}\right)\right| \leq b\left(\left|x_{i+2}\right|+\cdots+\left|x_{n}\right|\right), \\
& \left|g_{i}\left(\tilde{x}_{i+2}\right)\right| \leq b\left(\left|x_{i+2}\right|+\cdots+\left|x_{n}\right|\right) .
\end{aligned}
$$

Assumption 2. Without loss of generality, the sign of $d_{i}(t)$ is assumed to be positive, and there exist known positive constants $\lambda_{i}$ and $\mu_{i}$ such that, for any $t \in \mathbb{R}^{+}$and $i=1, \ldots, n$,

$$
0<\lambda_{i} \leq d_{i}(t) \leq \mu_{i} .
$$

Remark 4. From Assumption 1, the system investigated has an upper-triangular form. Due to the complex of uppertriangular system structure and the effect of stochastic noise, the stabilization of such systems is usually very difficult. In this paper, by using the low gain homogeneous domination approach, the state-feedback stabilization problem is investigated for the first time.

Remark 5. By Assumption 2, we know that $d_{i}(t)$ s are timevarying control coefficients; how to effectively deal with them in the design process is nontrivial work.

Firstly, introduce the following coordinate transformation:

$$
z_{i}=\frac{x_{i}}{\varepsilon^{i-1}}, \quad v=\frac{u}{\varepsilon^{n}}, \quad i=1, \ldots, n
$$


where $0<\varepsilon<1$ is a parameter to be designed. System (1) can be rewritten as

$$
\begin{gathered}
d z_{1}=\left(\varepsilon d_{1}(t) z_{2}+\bar{f}_{1}\left(\widetilde{z}_{3}\right)\right) d t+\bar{g}_{1}^{T}\left(\widetilde{z}_{3}\right) d \omega, \\
d z_{2}=\left(\varepsilon d_{2}(t) z_{3}+\bar{f}_{2}\left(\widetilde{z}_{4}\right)\right) d t+\bar{g}_{2}^{T}\left(\widetilde{z}_{4}\right) d \omega, \\
\vdots \\
d z_{n-2}=\left(\varepsilon d_{n-2}(t) z_{n-1}+\bar{f}_{n-2}\left(\widetilde{z}_{n}\right)\right) d t+\bar{g}_{n-2}^{T}\left(\widetilde{z}_{n}\right) d \omega, \\
d z_{n-1}=\varepsilon d_{n-1}(t) z_{n} d t, \\
d z_{n}=\varepsilon d_{n}(t) v d t,
\end{gathered}
$$

where $\bar{f}_{i}\left(\widetilde{z}_{i+2}\right)=f_{i}\left(\widetilde{x}_{i+2}\right) / \varepsilon^{i-1}, \bar{g}_{i}\left(\widetilde{z}_{i+2}\right)=g_{i}\left(\widetilde{x}_{i+2}\right) / \varepsilon^{i-1}$.

The nominal system for $(8)$ is

$$
\begin{gathered}
d z_{1}=d_{1}(t) z_{2}, \\
d z_{2}=d_{2}(t) z_{3}, \\
\vdots \\
d z_{n-2}=d_{n-2}(t) z_{n-1}, \\
d z_{n-1}=d_{n-1}(t) z_{n} d t, \\
d z_{n}=d_{n}(t) v d t .
\end{gathered}
$$

Theorem 6. For nominal system (9), with Assumption 2, one can design a stabilizing state-feedback controller to guarantee that

(1) the closed-loop system has an almost surely unique solution on $[0, \infty)$;

(2) the equilibrium of the closed-loop system is GAS in probability.

Proof. The controller design process proceeds step by step.

Step 1. Defining $\xi_{1}=z_{1}$ and choosing $V_{1}=(1 / 4) z_{1}^{4}$, from (9), it follows that

$$
\mathscr{L} V_{1} \leq d_{1}(t) z_{1}^{3} z_{2}
$$

Suppose that $z_{2}^{*}=-z_{1} \alpha_{1}=-\xi_{1} \alpha_{1}$, where $\alpha_{1} \geq 0$ is a constant to be chosen. Thus, by Assumption 2, we have

$$
d_{1}(t) z_{1}^{3} z_{2}^{*} \leq \lambda_{1} z_{1}^{3} z_{2}^{*} \leq 0 .
$$

By (10) and (11), one gets

$$
\mathscr{L} V_{1} \leq \lambda_{1} z_{1}^{3} z_{2}^{*}+d_{1}(t) z_{1}^{3}\left(z_{2}-z_{2}^{*}\right) .
$$

Choosing the virtual smooth control $z_{2}^{*}$ as

$$
z_{2}^{*}=-\frac{n}{\lambda_{1}} \xi_{1} \triangleq-\xi_{1} \alpha_{1}
$$

which substitutes into (12), yields

$$
\mathscr{L} V_{1} \leq-n \xi_{1}^{4}+d_{1}(t) z_{1}^{3}\left(z_{2}-z_{2}^{*}\right) .
$$

Deductive Step. Assume that, at step $k-1$, there are a $\mathscr{C}^{2}$, proper and positive definite Lyapunov function $V_{k-1}$, and the virtual controllers $z_{j}^{*}$ defined by

$$
\begin{gathered}
z_{1}^{*}=0, \quad \xi_{1}=z_{1}-z_{1}^{*}, \\
z_{2}^{*}=-\xi_{1} \alpha_{1}, \quad \xi_{2}=z_{2}-z_{2}^{*}, \\
\vdots \\
z_{k}^{*}=-\xi_{k-1} \alpha_{k-1}, \quad \xi_{k}=z_{k}-z_{k}^{*},
\end{gathered}
$$

where $\alpha_{i} \geq 0,1 \leq i \leq k-1$, are positive constants, such that

$$
\mathscr{L} V_{k-1}\left(\bar{z}_{k-1}\right) \leq-(n-k+2) \sum_{i=1}^{k-1} \xi_{i}^{4}+d_{k-1}(t) \xi_{k-1}^{3}\left(z_{k}-z_{k}^{*}\right)
$$

where $\bar{z}_{k-1}=\left(z_{1}, \ldots, z_{k-1}\right)^{T}$. To complete the induction, at the $k$ th step, one can choose the following Lyapunov function:

$$
V_{k}\left(\bar{z}_{k}\right)=V_{k-1}\left(\bar{z}_{k-1}\right)+\frac{1}{4} \xi_{k}^{4}
$$

where $\bar{z}_{k}=\left(z_{1}, \ldots, z_{k}\right)^{T}$.

By (15)-(17), one has

$$
\begin{aligned}
\mathscr{L} V_{k}\left(\bar{z}_{k}\right) \leq & -(n-k+2) \sum_{i=1}^{k-1} \xi_{i}^{4}+d_{k-1}(t) \xi_{k-1}^{3} \xi_{k} \\
& +\xi_{k}^{3}\left(d_{k}(t) z_{k+1}-\sum_{i=1}^{k-1} \frac{\partial z_{k}^{*}}{\partial z_{i}} d_{i}(t) z_{i+1}\right) .
\end{aligned}
$$

By using Young's inequality and Assumption 2, one has

$$
\begin{gathered}
d_{k-1}(t) \xi_{k-1}^{3} \xi_{k} \leq \frac{1}{2} \xi_{k-1}^{4}+c_{k} \xi_{k}^{4}, \\
-\xi_{k}^{3} \sum_{i=1}^{k-1} \frac{\partial z_{k}^{*}}{\partial z_{i}} d_{i}(t) z_{i+1} \leq c_{k 1}\left|\xi_{k}\right|^{3} \sum_{i=1}^{k}\left|z_{i}\right| \leq \frac{1}{2} \sum_{i=1}^{k-1} \xi_{i}^{4}+\widehat{c}_{k} \xi_{k}^{4},
\end{gathered}
$$

where $c_{k}>0, c_{k 1}>0$, and $\widehat{c}_{k}>0$ are constants. Suppose that

$$
z_{k+1}^{*}=-\xi_{k} \alpha_{k},
$$

where $\alpha_{k} \geq 0$ is a constant to be chosen. Then, by Assumption 2, one has

$$
d_{k}(t) \xi_{k}^{3} z_{k+1}^{*} \leq \lambda_{k} \xi_{k}^{3} z_{k+1}^{*} .
$$

Substituting (19) and (21) into (18) yields

$$
\begin{aligned}
\mathscr{L} V_{k}\left(\bar{z}_{k}\right) \leq & -(n-k+1) \sum_{i=1}^{k-1} \xi_{i}^{4}+d_{k}(t) \xi_{k}^{3}\left(z_{k+1}-z_{k+1}^{*}\right) \\
& +\lambda_{k} \xi_{k}^{3} z_{k+1}^{*}+\left(c_{k}+\widehat{c}_{k}\right) \xi_{k}^{4} .
\end{aligned}
$$


Choosing the virtual smooth control

$$
z_{k+1}^{*}=-\frac{1}{\lambda_{k}}\left(n-k+1+c_{k}+\widehat{c}_{k}\right) \xi_{k} \triangleq-\xi_{k} \alpha_{k},
$$

which substitutes into (22), yields

$$
\mathscr{L} V_{k}\left(\bar{z}_{k}\right) \leq-(n-k+1) \sum_{i=1}^{k} \xi_{i}^{4}+d_{k}(t) \xi_{k}^{3}\left(z_{k+1}-z_{k+1}^{*}\right)
$$

Step $n$. By choosing the actual control law

$$
v=-\xi_{n} \alpha_{n}
$$

where $\alpha_{n} \geq 0$ is a constant and $\xi_{n}=x_{n}-x_{n}^{*}$, one gets

$$
\mathscr{L} V_{n}\left(\bar{z}_{n}\right) \leq-\sum_{i=1}^{n} \xi_{i}^{4}
$$

where

$$
V_{n}\left(\bar{z}_{n}\right)=V_{n-1}\left(\bar{z}_{n-1}\right)+\frac{1}{4} \xi_{n}^{4}
$$

Finally, based on (26) and (27), by Lemma 3, one immediately gets the conclusion.

Now, we are in a position to get the main results of this paper.

Theorem 7. If Assumptions 1 and 2 hold for the uppertriangular stochastic nonlinear systems (1), with the coordinate transformation (7), by appropriately choosing the parameter $0<\varepsilon<1$, then, under the state-feedback controller (25), one has the following:

(1) the closed-loop system has an almost surely unique solution on $[0, \infty)$;

(2) the equilibrium of the closed-loop system is GAS in probability.

Proof. For system (8), with the state-feedback controller (25) and Lyapunov function (27), one has

$$
\mathscr{L} V_{n}\left(\bar{z}_{n}\right) \leq-\varepsilon \sum_{i=1}^{n} \xi_{i}^{4}+\sum_{i=1}^{n} \frac{\partial V_{n}}{\partial z_{i}} \bar{f}_{i}\left(\tilde{z}_{i+2}\right)+\frac{1}{2} \operatorname{Tr}\left\{G \frac{\partial^{2} V_{n}}{\partial z^{2}} G^{T}\right\},
$$

where $z=\left(z_{1}, \ldots, z_{n}\right)^{T}, G=\left(\bar{g}_{1}, \ldots, \bar{g}_{n-2}, 0,0\right)$. From (15) and (27), one has

$$
V_{n}\left(\bar{z}_{n}\right)=\frac{1}{4} \sum_{i=1}^{n} \xi_{i}^{4}=\frac{1}{4} \sum_{i=1}^{n}\left(z_{i}+c_{i, i-1} z_{i-1}+\cdots+c_{i, 1} z_{1}\right)^{4},
$$

where $c_{i, j}, j=1, \ldots, i-1$, are constants. By (7), (15) and Assumption 1, one can get

$$
\begin{aligned}
\left|\tilde{f}_{i}\left(\widetilde{z}_{i+2}\right)\right| & =\left|\frac{f_{i}\left(\tilde{x}_{i+2}\right)}{\varepsilon^{i-1}}\right| \leq b \varepsilon^{2} \sum_{j=i+2}^{n}\left|z_{j}\right| \\
& \leq b \varepsilon^{2} \sum_{j=i+2}^{n}\left(\left|\xi_{j}\right|+\alpha_{j-1}\left|\xi_{j-1}\right|\right) .
\end{aligned}
$$

By Young's inequality, using (29) and (30), one has

$$
\sum_{i=1}^{n} \frac{\partial V_{n}}{\partial z_{i}} \bar{f}_{i}\left(\widetilde{z}_{i+2}\right) \leq b_{f} \varepsilon^{2} \sum_{i=1}^{n} \xi_{i}^{4}
$$

Similarly, one can prove that

$$
\frac{1}{2} \operatorname{Tr}\left\{G \frac{\partial^{2} V_{n}}{\partial z^{2}} G^{T}\right\} \leq b_{g} \varepsilon^{2} \sum_{i=1}^{n} \xi_{i}^{4}
$$

Substituting (31) and (32) into (28), one has

$$
\begin{aligned}
\left.\mathscr{L} V_{n}\left(\bar{z}_{n}\right)\right|_{(8)} \leq & -\varepsilon \sum_{i=1}^{n} \xi_{i}^{4}+\left(b_{f}+b_{g}\right) \varepsilon^{2} \sum_{i=1}^{n} \xi_{i}^{4} \\
& =-\varepsilon\left(1-\left(b_{f}+b_{g}\right) \varepsilon\right) \sum_{i=1}^{n} \xi_{i}^{4} .
\end{aligned}
$$

By choosing $0<\varepsilon<1$ appropriately, (33) can be written as

$$
\left.\mathscr{L} V_{n}\left(\bar{z}_{n}\right)\right|_{(8)} \leq-c_{0} \sum_{i=1}^{n} \xi_{i}^{4}
$$

where $c_{0}>0$ is a constant.

By (34) and the coordinate transformation (7), using Lemma 3, the conclusions hold.

Remark 8. Theorems 6 and 7 provide us a new perspective to deal with the state-feedback control problem for uppertriangular stochastic nonlinear systems with time-varying coefficients. The main technical obstacle in the Lyapunov design for stochastic upper-triangular systems is that Itô stochastic differentiation involves not only the gradient but also the higher order Hessian term. The traditional design methods are invalid to deal with these terms. However, with the design methodology provided in Theorems 6 and 7, there is no need to estimate the bounds of drift and diffusion terms step by step. Based on this technique, a homogeneous nonlinear controller for the nominal nonlinear system is firstly constructed. Then we will design a scaled controller which can effectively dominate the drift and diffusion terms by taking advantage of the homogenous structure of the controller.

\section{A Simulation Example}

Consider the following system:

$$
\begin{gathered}
d x_{1}=\left(\left(2-\sin ^{2} t\right) x_{2}+x_{3} \sin x_{3}\right) d t+x_{3} \cos x_{3} d \omega, \\
d x_{2}=(2-\cos t) x_{3} d t \\
d x_{3}=\left(1+\sin ^{2} t\right) u d t .
\end{gathered}
$$

Obviously, Assumptions 1 and 2 hold.

Introduce the following coordinate transformation:

$$
z_{1}=x_{1}, \quad z_{2}=\frac{x_{2}}{\varepsilon}, \quad z_{3}=\frac{x_{3}}{\varepsilon^{2}}, \quad v=\frac{u}{\varepsilon^{3}},
$$



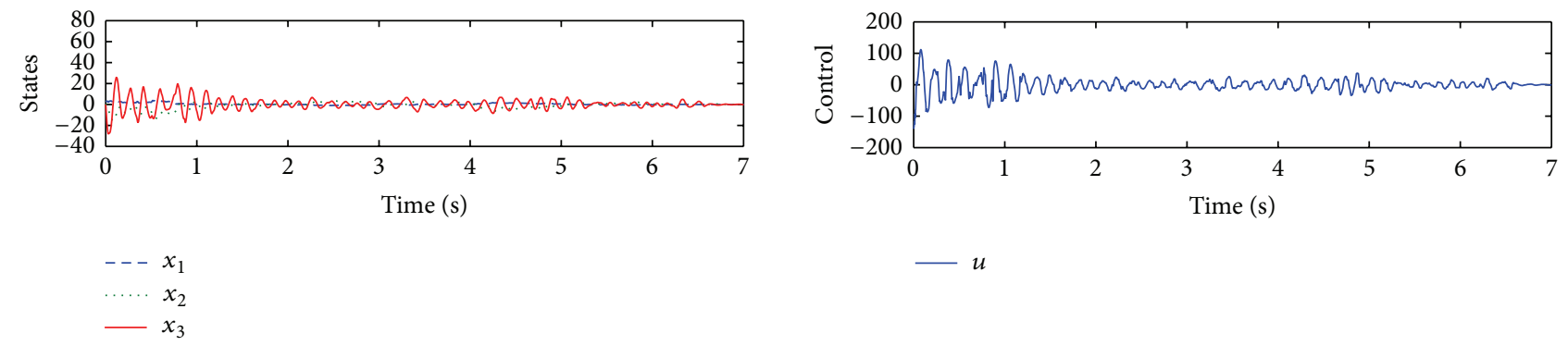

FIGURE 1: The response of closed-loop system (35)-(38).

where $0<\varepsilon<1$ is a design parameter. Then (35) can be written as

$$
\begin{gathered}
d z_{1}=\left(\varepsilon\left(2-\sin ^{2} t\right) z_{2}+\varepsilon^{2} z_{3} \sin ^{2}\left(\varepsilon^{2} z_{3}\right)\right) d t \\
+\varepsilon^{2} z_{3} \cos \left(\varepsilon^{2} z_{3}\right) d \omega, \\
d z_{2}=\varepsilon(2-\cos t) z_{3} d t \\
d z_{3}=\varepsilon\left(1+\sin ^{2} t\right) v d t .
\end{gathered}
$$

By following the design procedure in Section 3, one gets

$$
v\left(z_{1}, z_{2}, z_{3}\right)=-1310\left(372 z_{1}+124 z_{2}+z_{3}\right) \text {. }
$$

By choosing $\varepsilon=0.001$, with the initial values $z_{1}(0)=$ $3, z_{2}(0)=2$, and $z_{3}(0)=5$, Figure 1 gives the system response of the closed-loop system consisting of (35)-(38), from which the efficiency of the tracking controller is demonstrated.

\section{Concluding Remarks}

For a class of upper-triangular stochastic nonlinear systems with time-varying control coefficients, this paper investigates the state-feedback stabilization problem. The designed controller can guarantee that the closed-loop system has a unique solution and the closed-loop system can be proved to be GAS in probability.

There are many related problems to be investigated, for example, how to generalize the result in this paper to more general stochastic upper-triangular nonlinear systems.

\section{Conflict of Interests}

The authors declare that there is no conflict of interests regarding the publication of this paper.

\section{Acknowledgments}

This work is supported by National Natural Science Foundation (NNSF) of China under Grant nos. 61104128, 61174097; China Postdoctoral Science Foundation under Grant nos. 2012M520418, 2013T60185; Promotive Research Fund for Excellent Young and Middle-Aged Scientists of Shandong Province under Grant no. BS2013DX001; School Research Fund of Ludong University under Grant no. LY2012014.

\section{References}

[1] Z. Pan and T. Başar, "Adaptive controller design for tracking and disturbance attenuation in parametric strict-feedback nonlinear systems," IEEE Transactions on Automatic Control, vol. 43, no. 8, pp. 1066-1083, 1998.

[2] Z. Pan and T. Başar, "Backstepping controller design for nonlinear stochastic systems under a risk-sensitive cost criterion," SIAM Journal on Control and Optimization, vol. 37, no. 3, pp. 957-995, 1999.

[3] Z. Pan, K. Ezal, A. J. Krener, and P. V. Kokotović, "Backstepping design with local optimality matching," IEEE Transactions on Automatic Control, vol. 46, no. 7, pp. 1014-1027, 2001.

[4] H. Deng and M. Krstić, "Stochastic nonlinear stabilization. I. A backstepping design,” Systems \& Control Letters, vol. 32, no. 3, pp. 143-150, 1997.

[5] M. Krstić and H. Deng, Stabilization of Uncertain Nonlinear Systems, Springer, New York, NY, USA, 1998.

[6] H. Deng and M. Krstić, "Output-feedback stochastic nonlinear stabilization," IEEE Transactions on Automatic Control, vol. 44, no. 2, pp. 328-333, 1999.

[7] H. Deng and M. Krstić, "Output-feedback stabilization of stochastic nonlinear systems driven by noise of unknown covariance," Systems \& Control Letters, vol. 39, no. 3, pp. 173$182,2000$.

[8] H. Deng, M. Krstić, and R. J. Williams, "Stabilization of stochastic nonlinear systems driven by noise of unknown covariance," IEEE Transactions on Automatic Control, vol. 46, no. 8, pp. 12371253, 2001.

[9] Z.-J. Wu, X.-J. Xie, and S.-Y. Zhang, "Adaptive backstepping controller design using stochastic small-gain theorem," Automatica, vol. 43, no. 4, pp. 608-620, 2007.

[10] Z.-J. Wu, X.-J. Xie, and S.-Y. Zhang, "Stochastic adaptive backstepping controller design by introducing dynamic signal and changing supply function," International Journal of Control, vol. 79, no. 12, pp. 1635-1646, 2006.

[11] X. Yu and X.-J. Xie, "Output feedback regulation of stochastic nonlinear systems with stochastic iISS inverse dynamics," IEEE Transactions on Automatic Control, vol. 55, no. 2, pp. 304-320, 2010.

[12] X. Yu, X.-J. Xie, and N. Duan, "Small-gain control method for stochastic nonlinear systems with stochastic iISS inverse dynamics," Automatica, vol. 46, no. 11, pp. 1790-1798, 2010.

[13] W. Li, Y. Jing, and S. Zhang, "Adaptive state-feedback stabilization for a large class of high-order stochastic nonlinear systems," Automatica, vol. 47, no. 4, pp. 819-828, 2011. 
[14] W. Li, X. Liu, and S. Zhang, "Further results on adaptive state-feedback stabilization for stochastic high-order nonlinear systems," Automatica, vol. 48, no. 8, pp. 1667-1675, 2012.

[15] X.-J. Xie and W.-Q. Li, "Output-feedback control of a class of high-order stochastic nonlinear systems," International Journal of Control, vol. 82, no. 9, pp. 1692-1705, 2009.

[16] W. Li, Y. Jing, and S. Zhang, "Output-feedback stabilization for stochastic nonlinear systems whose linearizations are not stabilizable," Automatica, vol. 46, no. 4, pp. 752-760, 2010.

[17] A. R. Teel, "Global stabilization and restricted tracking for multiple integrators with bounded controls," Systems \& Control Letters, vol. 18, no. 3, pp. 165-171, 1992.

[18] F. Mazenc, "Stabilization of feedforward systems approximated by a non-linear chain of integrators," Systems \& Control Letters, vol. 32, no. 4, pp. 223-229, 1997.

[19] F. Mazenc and L. Praly, "Adding integrations, saturated controls, and stabilization for feedforward systems," IEEE Transactions on Automatic Control, vol. 41, no. 11, pp. 1559-1578, 1996.

[20] X. Ye, "Universal stabilization of feedforward nonlinear systems," Automatica, vol. 39, no. 1, pp. 141-147, 2003. 


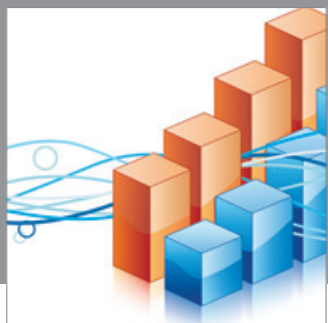

Advances in

Operations Research

mansans

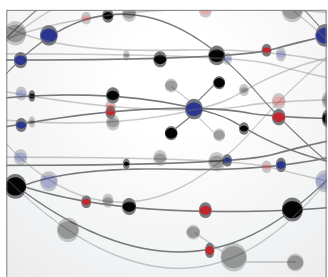

The Scientific World Journal
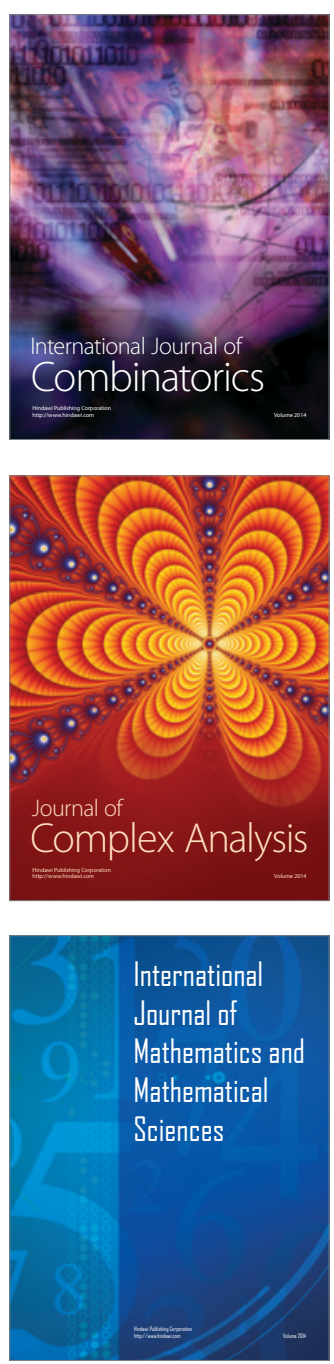
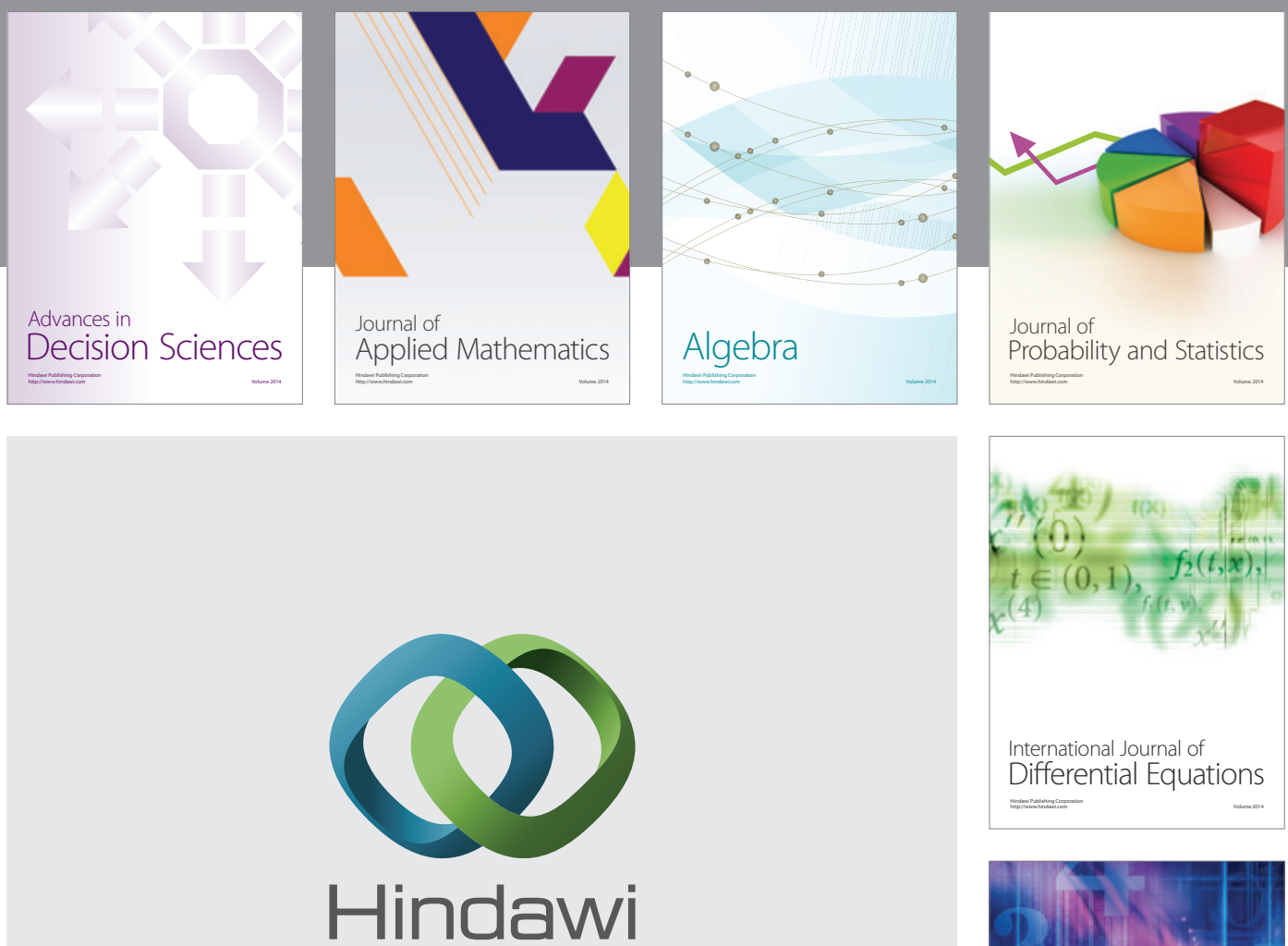

Submit your manuscripts at http://www.hindawi.com
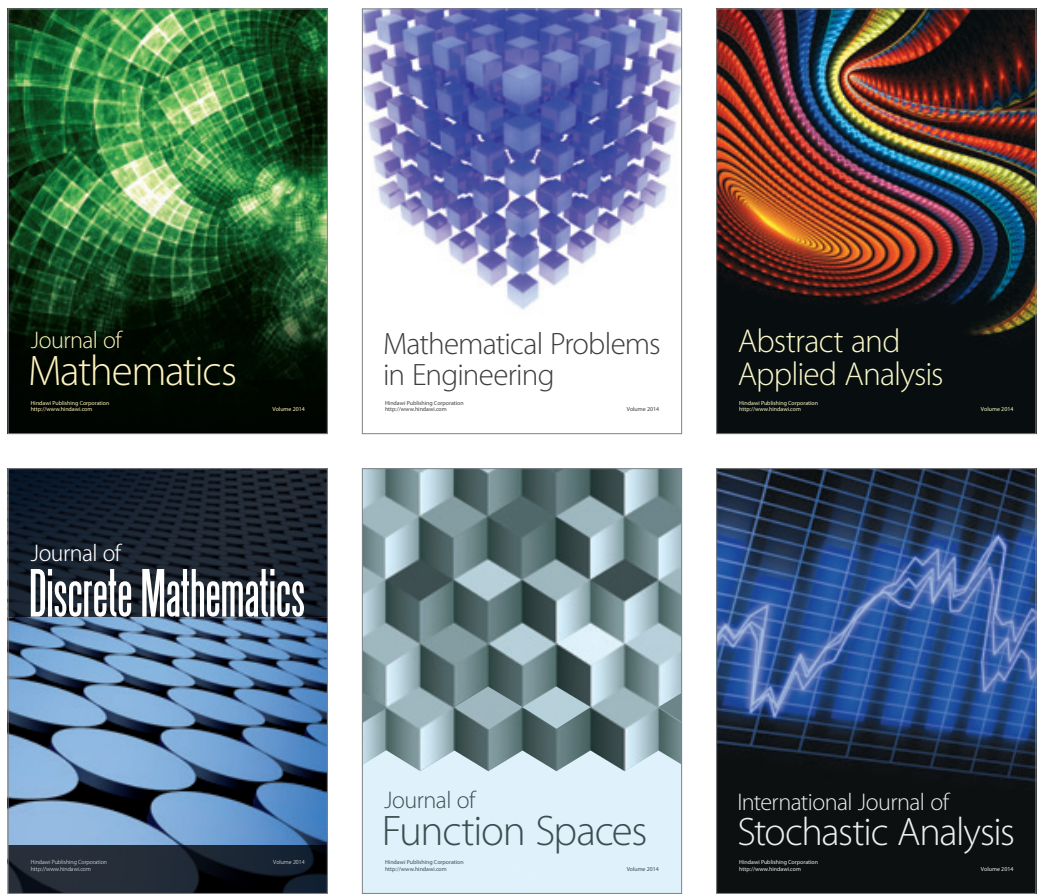

Journal of

Function Spaces

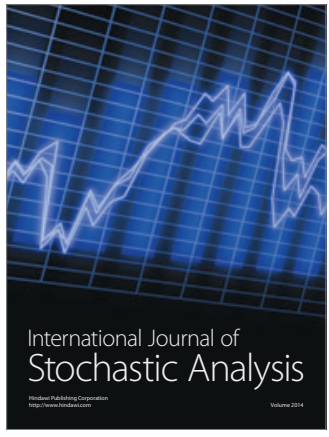

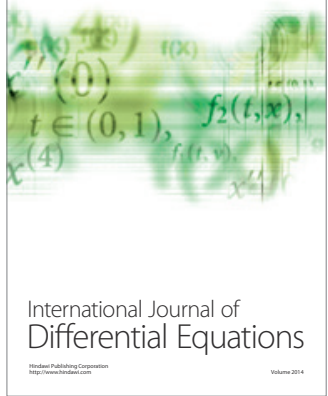
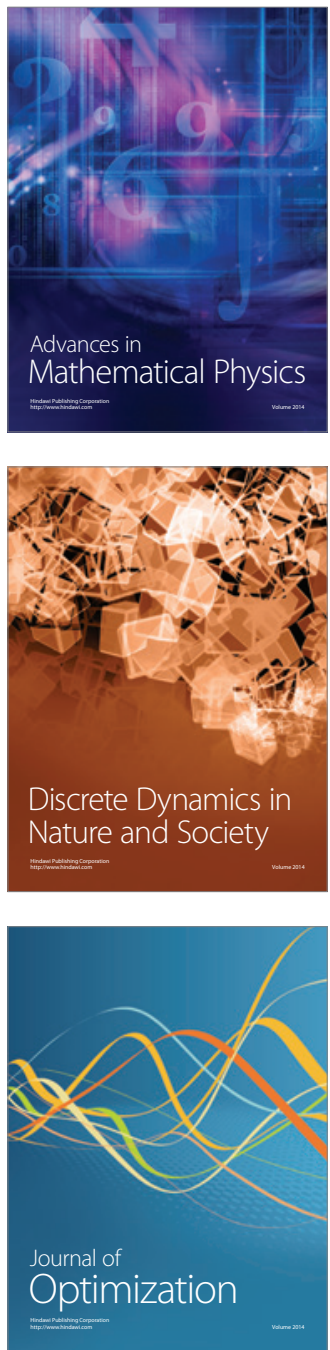